ARTICLE

Received 28 Aug 2014 | Accepted 3 Feb 2015 | Published 23 Mar $2015 \quad$ DOl: 10.1038/ncomms7569 OPEN

\title{
SINE transcription by RNA polymerase III is suppressed by histone methylation but not by DNA methylation
}

Dhaval Varshney ${ }^{1,2, \star}$, Jana Vavrova-Anderson ${ }^{1, \star}$, Andrew J. Oler ${ }^{3,4}$, Victoria H. Cowling ${ }^{2}$, Bradley R. Cairns ${ }^{3,4} \&$ Robert J. White 1,5

Short interspersed nuclear elements (SINEs), such as Alu, spread by retrotransposition, which requires their transcripts to be copied into DNA and then inserted into new chromosomal sites. This can lead to genetic damage through insertional mutagenesis and chromosomal rearrangements between non-allelic SINEs at distinct loci. SINE DNA is heavily methylated and this was thought to suppress its accessibility and transcription, thereby protecting against retrotransposition. Here we provide several lines of evidence that methylated SINE DNA is occupied by RNA polymerase III, including the use of high-throughput bisulphite sequencing of ChIP DNA. We find that loss of DNA methylation has little effect on accessibility of SINEs to transcription machinery or their expression in vivo. In contrast, a histone methyltransferase inhibitor selectively promotes SINE expression and occupancy by RNA polymerase III. The data suggest that methylation of histones rather than DNA plays a dominant role in suppressing SINE transcription.

\footnotetext{
${ }^{1}$ College of Medical, Veterinary and Life Sciences, University of Glasgow, Glasgow G12 8QQ, UK. ${ }^{2}$ The MRC Protein Phosphorylation Unit, University of Dundee, Dow Street, Dundee DD1 5EH, UK. ${ }^{3}$ Department of Oncological Sciences, Huntsman Cancer Institute, University of Utah School of Medicine, Salt Lake City, Utah 84112, USA. ${ }^{4}$ Howard Hughes Medical Institute, University of Utah School of Medicine, Salt Lake City, Utah 84112, USA. ${ }^{5}$ Department of Biology, University of York, York YO10 5DD, UK. * These authors contributed equally to this work. Correspondence and requests for materials should be addressed to D.V. (email: d.varshney@dundee.ac.uk) or to R.J.W. (email: bob.white@york.ac.uk).
} 
A striking feature of mammalian chromosomes is the large numbers of short interspersed nuclear elements (SINEs) interspersed among the genes. For example, the human genome carries $\sim 10^{6}$ copies of Alu SINEs that together account for $\sim 11 \%$ of total chromosomal DNA. Such SINEs have had a major impact on genomic evolution and place a burden on chromosomal stability through deletions and translocations arising by recombination between non-allelic loci. Indeed, SINEs have caused many instances of human genetic disease ${ }^{1,2}$, including $\alpha$-thalassaemia ${ }^{3}$, hypercholesterolaemia ${ }^{4}$ and neurofibromatosis ${ }^{5}$.

SINEs spread by retrotransposition, which requires their transcripts to be copied into DNA and then inserted into new chromosomal sites. SINE promoters direct transcription by RNA polymerase (pol) III, but expression is extremely weak. For example, the million Alu templates together produce only $\sim 10^{3}$ transcripts in HeLa cells, whereas the three $7 S L$ genes per genome produce $\sim 10^{6}$ transcripts using the same pol III transcription factors as $\mathrm{Alu}^{6}$. It was estimated that $\sim 99 \%$ of potentially active Alu SINEs with intact promoters may be silenced ${ }^{7}$. Such transcriptional repression is believed to involve packaging the SINEs into chromatin structures that deny access of transcription factors. This may be of great importance, because SINEs can exert boundary effects and regulate messenger RNA (mRNA) synthesis ${ }^{8}$. SINE transcript overexpression can be cytotoxic and cause an untreatable form of human blindness ${ }^{9}$.

SINE transcription is thought to be silenced through methylation of $\mathrm{CpG}$, an important mechanism of gene repression in mammals ${ }^{10,11}$. About 7 million of the total 30 million $\mathrm{CpG}$ sites in the human genome lie within Alu sequences ${ }^{12}$, with $\mathrm{CpG}$ densities ninefold above the average for the human genome in some subfamilies ${ }^{13}$. Heavy methylation is found at the majority of Alu and mouse SINEs ${ }^{14-16}$. That this contributes to transcriptional repression was suggested by an increase in Alu expression after treatment of HeLa cells with the DNA methylation inhibitor 5-azacytidine ${ }^{6}$. Furthermore, methylation of Alu DNA was found to repress its transcription in transient transfections and in vitro under some conditions ${ }^{13,15,17}$. Repression in vitro was relieved by miscellaneous methylated competitor DNA, suggesting that it is mediated by one or more trans-acting factors with sequence independent affinity for methylated DNA ${ }^{13}$. Examples of such methyl-CpG-binding proteins (MBPs) include MeCP2, MBD1 and MBD2, which operate primarily by directing assembly of repressive chromatin structures that are inaccessible to the transcription machinery ${ }^{18}$. Indeed, MeCP2 was found at Alu SINEs in human cells ${ }^{19}$.

Here we show that human and mouse SINE families are occupied by MeCP2, MBD1 and MBD2. However, SINE transcription is not enhanced by DNA demethylation and release of these MBPs. In contrast, pol III loading and expression of SINEs increases significantly when cells are treated with chaetocin, a selective inhibitor of SUV39 methyltransferases that methylates histone H3 on lysine 9 (H3K9). Endogenous SUV39 associates with SINEs and SINE induction by chaetocin correlates with loss of trimethylated $\mathrm{H} 3 \mathrm{~K} 9$. The data suggest that methylation of $\mathrm{H} 3 \mathrm{~K} 9$, rather than DNA, is primarily responsible for suppressing pol III-mediated transcription of genomic SINEs in the cells we have studied.

\section{Results}

MBPs target SINEs. Consistent with the model that SINEs are subject to MBP-mediated silencing, semiquantitative and quantitative chromatin immunoprecipitation (ChIP)-PCR assays detected MeCP2, MBD1 and MBD2 in HeLa cells at multiple Alu sites, chosen randomly from several chromosomes (Fig. 1a,b).
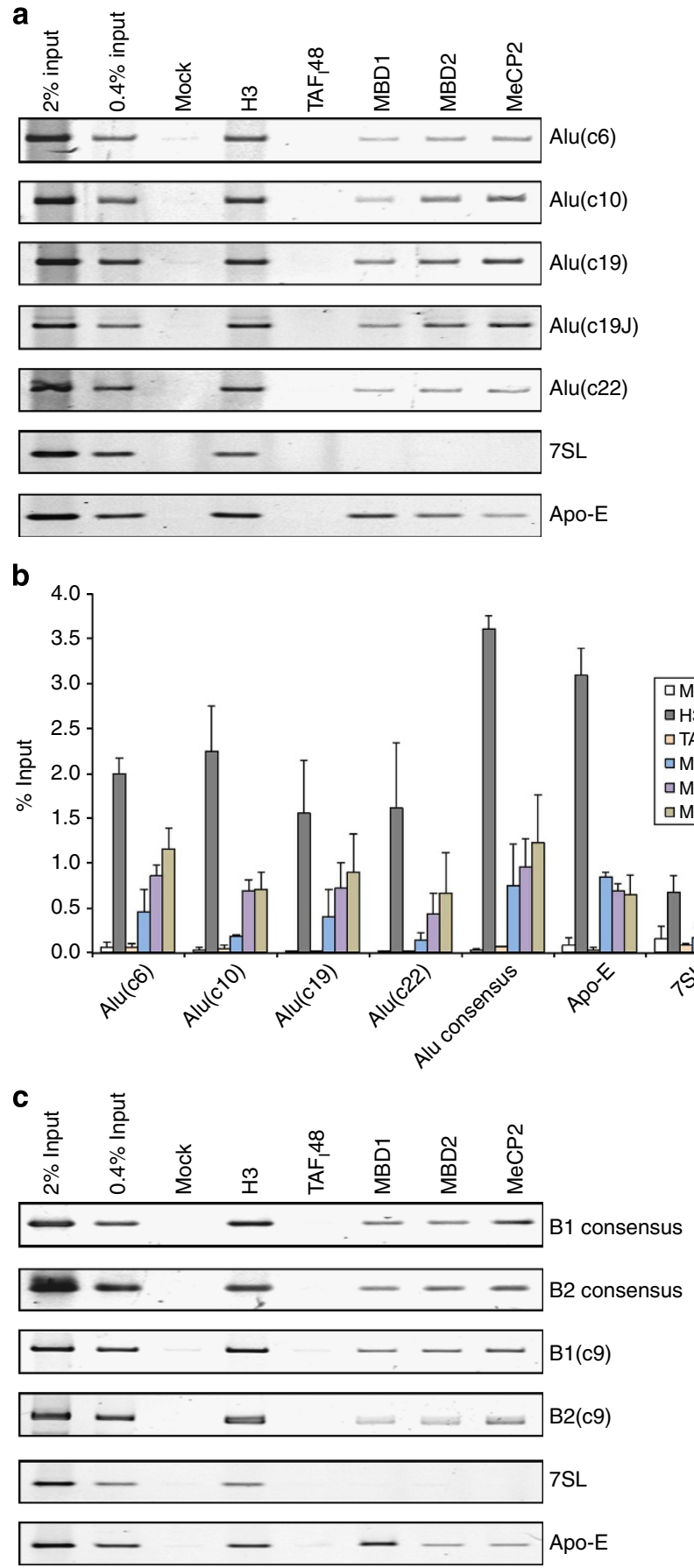

Figure 1 | SINEs provide binding sites for MBPs. (a) Semiquantitative ChIP assay in HeLa cells showing the binding of MBD1, MBD2 and MeCP2 at Alu loci from chromosomes 6, 10, 19 and 22, as well as 7SL and Apo-E loci. Alu19] is also from chromosome 19. ChIPs for histone $\mathrm{H} 3$ and $\mathrm{TAF}_{1} 48$ provide positive and negative controls, respectively. No antibody was used for the mock sample. (b) Mean \pm s.e.m. of the percentage input bound in two independent ChIP-quantitative PCR assays with HeLa cells and antibodies against histone $\mathrm{H} 3$, TAF 48 , MBD1, MBD2 and MeCP2, as indicated. No antibody was used for the mock samples. Amplifications were carried out using primers for the Apo- $E$ and $7 S L$ genes, Alu PV subfamily consensus sequence and individual Alus on chromosomes 6, 10, 19 and 22, as indicated. (c) Semiquantitative ChIP assay in A31 fibroblasts showing binding of MBD1, MBD2 and MeCP2 at B1 and B2 loci, as well as 7SL and Apo-E genes. B1 and B2 consensus primers match $\sim 10^{2}$ members of the B1 and B2 families, whereas B1(c9) and B2(c9) primers detect unique loci on chromosome 9. ChIPs for histone $\mathrm{H} 3$ and $\mathrm{TAF}_{1} 48$ provide positive and negative controls, respectively. 
These Alus have 7-13 CpGs each and include an example from chromosome 6 (c6) that is embedded in a cluster of SINEs, $\sim 5 \mathrm{~kb}$ from the nearest annotated pol II-transcribed gene. Binding was also detected using consensus primers that recognize $\sim 10^{3}$ members of an Alu subfamily. Signal intensity is comparable to that obtained with the pol II-dependent Apo-E gene, which is known to be silenced by $\mathrm{MBPs}^{20}$. Furthermore, binding is selective, as it was not observed at $7 S L$ genes, the ancestral progenitors of $\mathrm{Alu}^{21}$. In contrast to SINEs, 7SL sequences are predominantly unmethylated in genomic DNA.

In rodent genomes, the most abundant SINE families are B1 and B2, instead of Alu ${ }^{22}$. Whereas B1 resembles Alu, both having evolved from 7SL RNA, B2 is unrelated and derived from a transfer RNA (tRNA) ${ }^{21,23}$. Nevertheless, both B1 and B2 SINEs are occupied by MeCP2, MBD1 and MBD2 in mouse fibroblasts (Fig. 1c). In each case, clear binding was detected using primers that recognise familial consensus sequences, as well as with unique loci from chromosome 9 (c9). We conclude that abundant mouse and human SINEs are targeted by proteins that mediate transcriptional silencing directed by DNA methylation.

MBPs do not exclude pol III from SINEs. Despite the presence of MBPs, pol III was also detected at these SINEs, as were the pol III-specific transcription factors TFIIIB and TFIIIC (Fig. 2a,b). Specificity was confirmed by the lack of pol III, TFIIIB and TFIIIC at the pol II-dependent Apo-E gene. ChIP-quantitative PCR demonstrated that pol III detection at consensus Alu
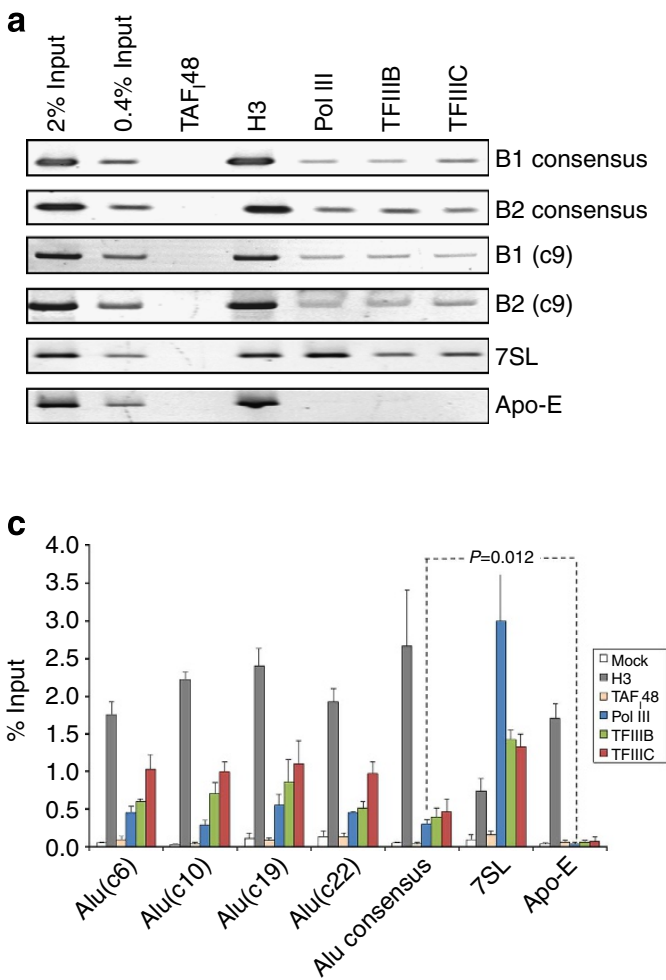

elements is approximately sixfold weaker than at the highly active $7 S L$ genes, but is nevertheless significantly above the background observed on the Apo-E gene or with a negative control antibody against $\mathrm{TAF}_{\mathrm{I}} 48$, a pol I-specific transcription factor (Fig. 2c). We conclude that chromosomal SINEs are more accessible to pol III and its associated transcription factors than had previously been thought.

The clear detection of both pol III and MBPs at these loci could be explained if a mixed cell population was present, with the same SINE occupied by MBPs in some cells and by pol III machinery in others. Sequential ChIP assays were used to test this hypothesis. DNA occupied by pol III was immunoprecipitated and the samples were then re-immunoprecipitated with antibodies to MeCP2, MBD1 and MBD2, as well as TFIIIB and pol III again as positive controls. For each MBP tested, Alu DNA was recovered at the levels well above background, indicating that all three MBPs can interact with a SINE at the same time as pol III (Fig. 2d). This was also the case for B1 and B2 SINEs and when TFIIIC was chromatin immunoprecipitated first instead of pol III (Supplementary Fig. 1).

Co-occupancy of MBPs with pol III and its associated factors suggests that the latter can bind to methylated DNA. This possibility is supported by ChIP-chop assays, in which DNA recovered from ChIP samples is subjected to restriction digestion at sequences of potential methylation ${ }^{24}$. As expected, Alu DNA associated with MBD2 or MeCP2 was digested more readily by the methylation-insensitive restriction enzyme MspI than by its methylation-sensitive isoschizomer HpaII (Supplementary Fig. 2).

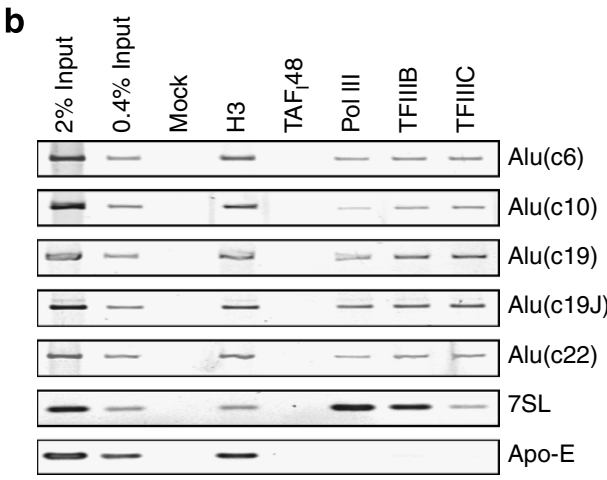

d

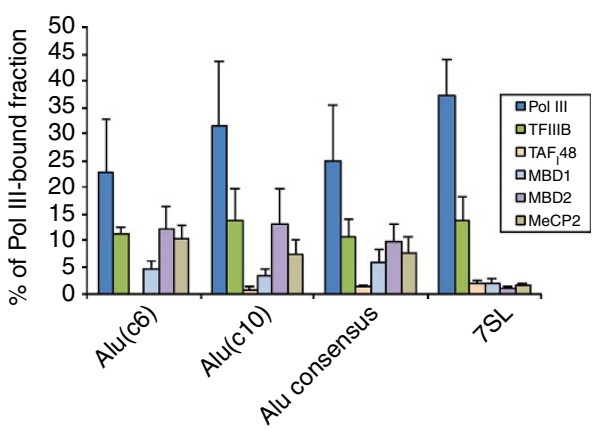

Figure 2 | Pol III co-occupies methylated SINEs with MBPs. (a) Semiquantitative ChIP assay in A31 fibroblasts showing specific binding of TFIIIB, TFIIIC and pol III to $B 1$ and $B 2$ loci, as well as 7SL, but not the Apo-E gene. Histone $\mathrm{H} 3$ and $T A F_{1} 48$ provide positive and negative controls, respectively. (b) Semiquantitative ChIP assay in HeLa cells showing occupancy of pol III, TFIIIB and TFIIIC at Alu loci from chromosomes 6, 10, 19 and 22, as well as 7SL and Apo-E genes. ChIPs for histone $\mathrm{H} 3$ and $\mathrm{TAF}_{1} 48$ provide positive and negative controls, respectively. No antibody was used for the mock sample. (c) Mean \pm s.e.m. of the percentage input bound in three independent ChIP-quantitative PCR ( $\mathrm{PPCR}$ ) assays in HeLa cells, of the indicated proteins at individual Alu loci from chromosomes 6, 10, 19 and 22, as well as 7SL and Apo-E loci and Alu PV subfamily consensus. ChIPs for histone H3 and TAF,48 provide positive and negative controls, respectively. No antibody was used for the mock samples. $P$ values are calculated by $t$-test. (d) Mean \pm s.e.m. of four independent sequential ChIP-qPCR assays in which DNA immunoprecipitated from HeLa cells using pol III antibody was reprecipitated using antibodies against pol III, TFIIIB, TAF,48 (negative control), MBD1, MBD2 and MeCP2, as indicated. No TAF,48 signal was detected on Alu(c6). 
The same was found for Alu DNA bound by pol III or TFIIIC, demonstrating that these proteins also interact in vivo with methylated SINE DNA.

Bisulphite sequencing of a pol III-bound Alu following ChIP demonstrated the high levels of CpG methylation (Supplementary Fig. 3). To examine this globally, we applied the recently reported genome-scale ChIP-bisulphite-sequencing (ChIP-BS-Seq) technique to assess DNA methylation status of pol III-bound DNA ${ }^{25}$. Using this method, we found that the methylation levels are comparable for input DNA and for Alu DNA cross-linked to pol III, showing that DNA methylation does not deter occupancy (Fig. 3a). This is the case for all Alu classes, including the relatively young AluY subclasses that are most active in retrotransposition. Indeed, the AluY SINEs generally show significantly higher DNA methylation than the older AluS and AluJ classes, and also higher pol III occupancy, although the latter does not reach statistical significance (Supplementary Data 1 provides the full data set). The AluYa5 subclass has the highest occupancy by pol III, despite being one of the most strongly methylated (Fig. 3b). Inverse correlation was not found between pol III occupancy and DNA methylation. Focusing on the B-block region, which provides the primary, high-affinity binding site for TFIIIC, comparison of the three main classes revealed CpG methylation of $55-65 \%$ for AluY, compared with $30-50 \%$ for AluS and $13 \%$ for AluJ, the oldest Alu class. It is striking that the $\mathrm{A}$ - and B-block promoter regions that direct transcription complex assembly contain $\mathrm{CpG}$ dinucleotides that are methylated in pol III-bound DNA at comparable levels to the input DNA (Fig. 3c; Supplementary Fig 4). Indeed, 12.9\% of Alu SINEs show statistically significant enrichment for CpG methylation in the pol III ChIP relative to the input DNA, whereas this is only the case for $0.4 \%$ of tRNA genes. Overall, Alu SINEs have much higher levels of CpG methylation than tRNA genes, irrespective of whether they are occupied by pol III (Fig. 3d). Collectively, the data indicate that template methylation does not prevent access of pol III to SINEs. Furthermore, pol III can be detected throughout the length of occupied SINEs (Supplementary Fig 5).

We tested the effect on pol III occupancy of treating cells with 5 -azacytidine at concentrations that elicit global genomic demethylation. The efficacy of the treatment was confirmed by
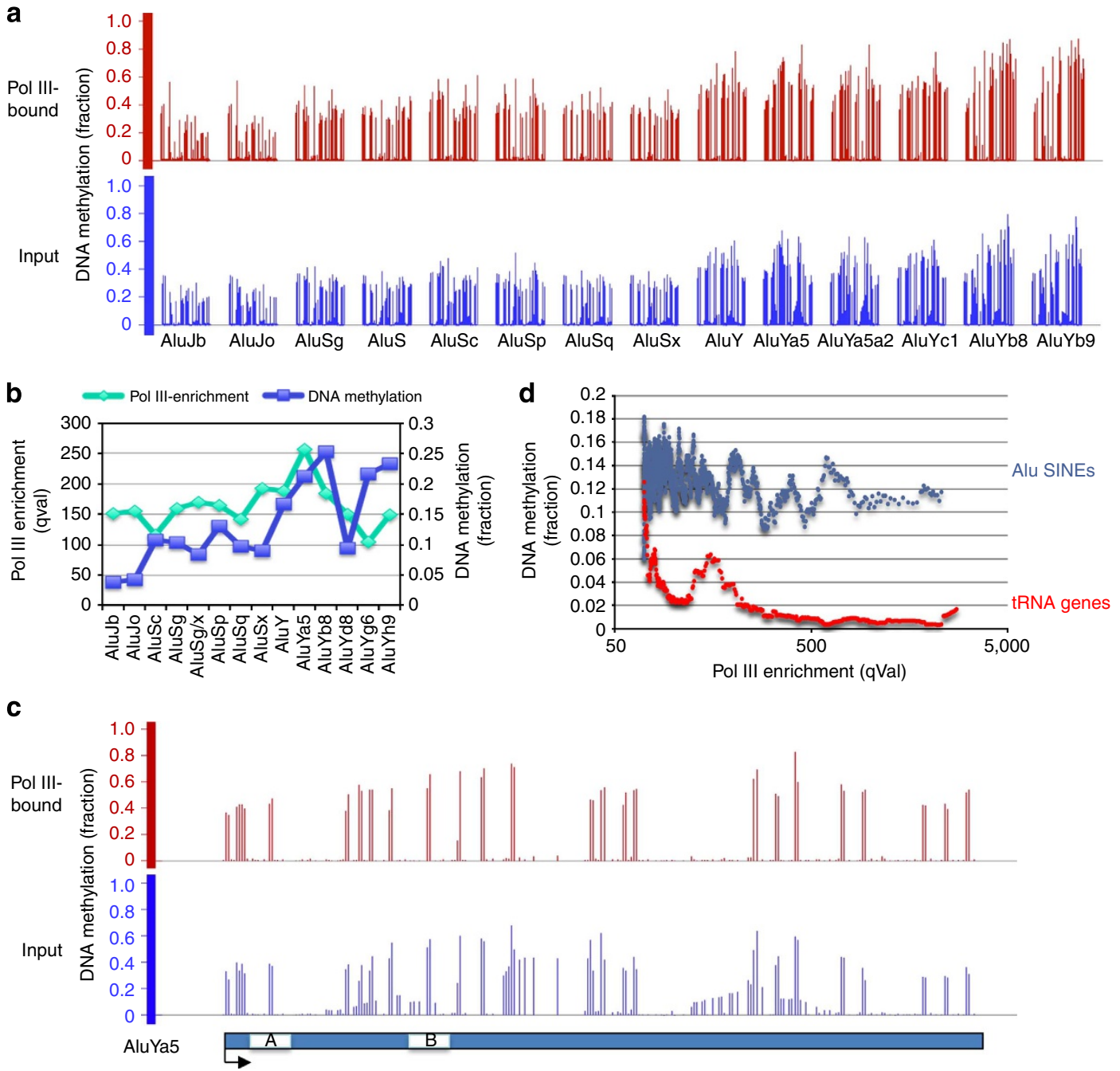

Figure 3 | Genome-wide methylation analysis of pol III-bound Alu DNA. (a) Comparison of the fraction of methylation of the indicated Alu subfamilies between genomic DNA (input) and pol III-bound DNA, as determined by ChIP-BS-Seq. The height of a vertical line indicates the fraction methylated at a particular C within the subfamily consensus sequence. (b) Genome-wide comparison of pol III occupancy and DNA methylation of Alu subfamilies. (c) Comparison of the extent of methylation of the AluYa5 subfamily between genomic DNA (input) and pol III-bound DNA. The height of a vertical line indicates the fraction methylated at a particular $\mathrm{C}$ within the subfamily consensus sequence. A- and B-block promoter elements are indicated. (d) Pol III occupancy plotted against DNA methylation for Alu SINEs (blue) and tRNA genes (red). Data are plotted as a moving average with 25 data points per window. 

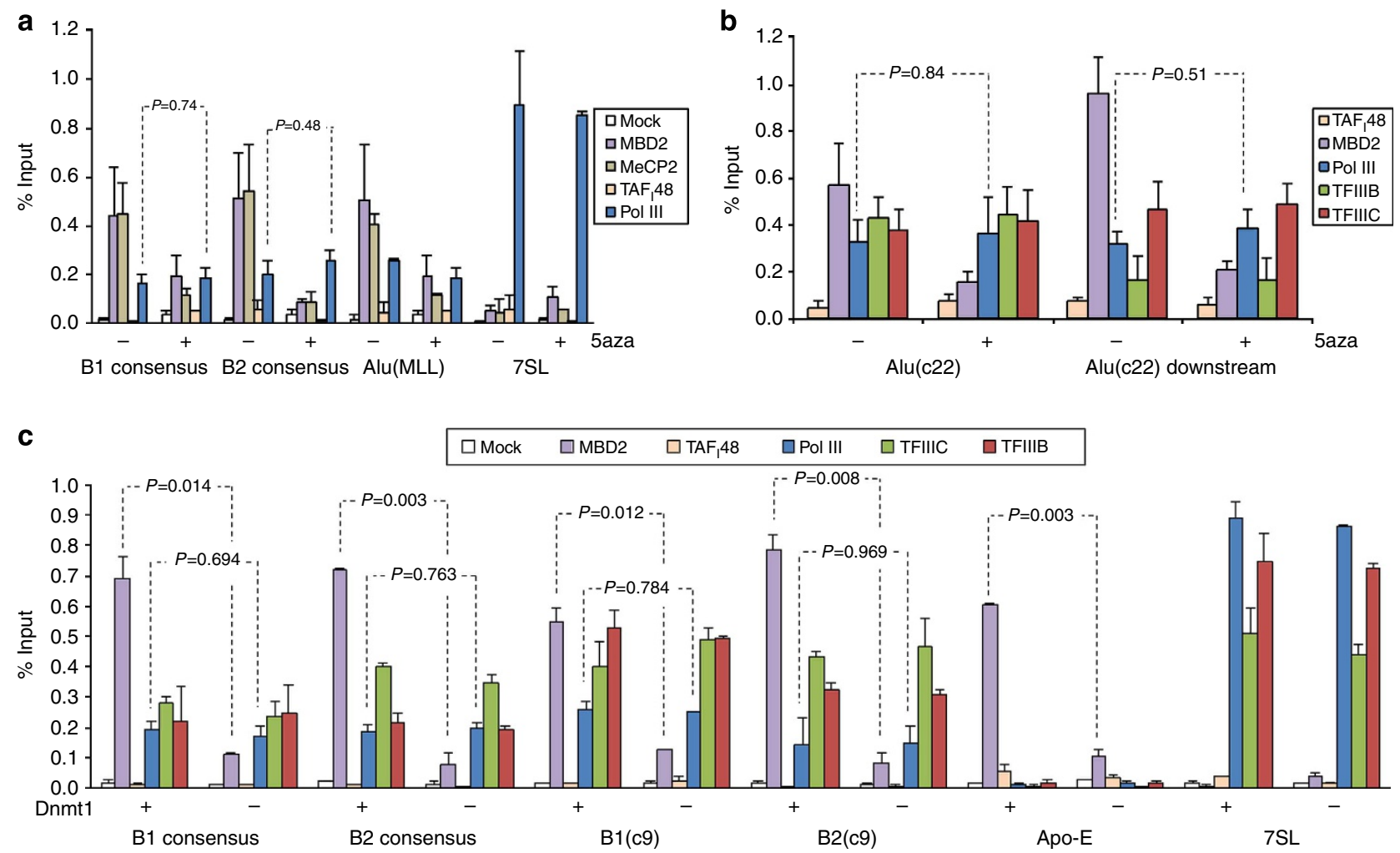

Figure 4 | DNA methylation does not prevent pol III occupancy of SINEs. (a) Percentage input bound in three independent ChIP-quantitative PCR (qPCR) assays with mouse ES cells treated for $16 \mathrm{~h}$ with $(+)$ or without $(-)$ 5-azacytidine, showing occupancy of MBD2, MeCP2 and pol III at $7 \mathrm{SL}$, B1 and B2 loci, as well as an Alu inserted onto chromosomes 14 and 17. ChIPs for TAF,48 and without antibody (mock) provide negative controls. (b) Percentage input bound in three independent ChIP-qPCR assays with HeLa cells treated for $72 \mathrm{~h}$ with $(+)$ or without $(-)$-azacytidine, showing the binding of MBD2, TFIIIB, TFIIIC and pol III to DNA centred over the body of Alu(c22) or $200 \mathrm{bp}$ downstream. The resolution of this assay is limited by the size of the genomic DNA fragments $(\sim 500 \mathrm{bp})$. (c) Percentage input bound in two independent ChIP-qPCR assays with matched Dnmt $1^{+/}+$and Dnmt1-/ - fibroblasts showing occupancy of MBD2, TFIIIB, TFIIIC and pol III at B1 and B2 loci, as well as 7SL and Apo-E genes. ChIPs for TAF,48 and without antibody (mock) provide negative controls. Error bars indicate s.e.m. and all $P$ values are calculated by $t$-test.

a clear decrease in MBP binding in vivo (Fig. 4a,b) and was confirmed in vitro following extraction of genomic DNA (Supplementary Fig. 6). However, pol III detection at Alu, B1 and B2 SINEs was not increased, providing further evidence that DNA methylation does not preclude access of pol III to SINEs (Fig. 4a,b). Detection of TFIIIB and TFIIIC at SINEs is also unaffected by 5 -azacytidine (Fig. $4 \mathrm{~b}$ ). To address the possibility that $\mathrm{CpG}$ demethylation might affect the distribution of pol III along its template, we included primers located downstream of an Alu. As expected, the signal for TFIIIB is weaker using the downstream primers, whereas robust signals from pol III and TFIIIC are maintained; this is consistent with the established localization of these proteins ${ }^{26}$. As with other primer sets, the pol III signal with the downstream primer set was not significantly affected by 5 -azacytidine (Fig. 4b). Although the shortness of SINEs limits the study of polymerase distribution using this technology, the available data do not support the possibility that demethylation alters pol III positioning along SINE DNA.

We also found comparable levels of SINE occupancy by pol III between fibroblasts with or without targeted disruption of the gene encoding Dnmt1 (Fig. 4c), despite the fact that the Dnmt1knockout cells have $<5 \%$ of the normal level of DNA methylation $^{27}$. The ChIP signals from TFIIIB and TFIIIC on SINEs are also comparable in the presence or absence of Dnmt1. Although expression of MBD2 and MeCP2 is undiminished in the knockout cells, their binding to B1 and B2 loci is significantly compromised (Fig. 4c; Supplementary Fig. 7). This observation excludes the unlikely possibility that methylation of B1 or B2 DNA is immune to loss of Dnmt1. We conclude that pol III and its associated transcription factors are not prevented from accessing SINEs in vivo by DNA methylation or the presence of various MBPs.

SINE repression does not rely on DNA methylation. Dnmt1knockout fibroblasts show little or no increase in B1 or B2 RNA (Fig. 5a), despite robust induction of the Apo-E and $p 53 B P 2$ genes, both of which are subject to methylation-dependent silencing ${ }^{20,28}$. This is consistent with a previous report that the B1 transcript levels are unchanged relative to wild type in Dnmt $1^{-/-}$or Dnmt3ab-/- embryonic stem (ES) cells, in which CpG methylation of B1 DNA is reduced by approximately two- to fourfold ${ }^{29}$.

Because SINEs are often located within introns and untranslated regions of protein-coding genes, SINE sequences are found within many longer pol II-derived RNAs. As this can complicate interpretation, we applied $\alpha$-amanitin at a concentration that inhibits pol II while allowing transcription by pol III. Again we found no evidence that B1 or B2 expression is elevated in Dnmt $1^{-1}$ - fibroblasts relative to wild type (Supplementary Fig. 8). This is consistent with the unchanged occupancy by pol III, TFIIIB and TFIIIC, as determined by ChIP (Fig. 4c).

As knockout cells have the potential to adapt to permanent loss of a component, we also used 5-azacytidine to promote DNA demethylation in wild-type cells. This caused no increase in the 


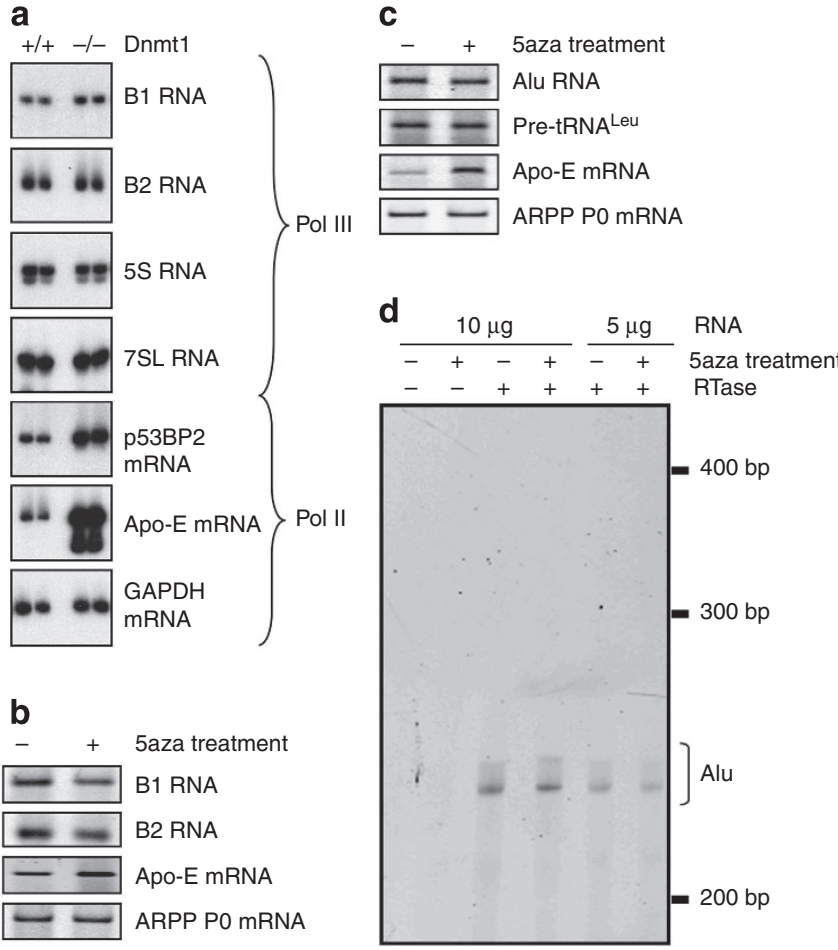

Figure 5 | SINE expression is not stimulated by loss of DNA methylation.

(a) Analysis by semiquantitative RT-PCR of expression levels of the indicated transcripts in matched Dnmt1 ${ }^{+} /+$and Dnmt1-/- fibroblasts. Duplicate samples are shown for both cell types. Apo-E and p53BP2

mRNAs provide controls that have been documented as being suppressed by DNA methylation. GAPDH mRNA provides a loading control.

(b) Analysis by semiquantitative RT-PCR of expression levels of the indicated transcripts in mouse ES cells treated for $16 \mathrm{~h}$ with $(+)$ or without (-) 5-azacytidine. Apo-E mRNA provides a control that has been documented as being inhibited by DNA methylation. ARPP PO mRNA provides a loading control. (c) Analysis by semiquantitative RT-PCR of expression levels of the indicated transcripts in HeLa cells treated for $72 \mathrm{~h}$ with 5-azacytidine. Apo-E mRNA provides a control that has been documented as being inhibited by DNA methylation. ARPP PO mRNA provides a loading control. (d) Analysis by primer extension of Alu transcripts in the RNA from Fig. 5c. Bracket indicates $\sim 240$ bp products that initiate at the principle pol III start site of Alu. Reverse transcriptase was omitted from the reactions in lanes 1 and 2 . To confirm that the assay was not saturated, raising the amount of template RNA from 5 (lanes 5 and 6) to $10 \mu$ g (lanes 3 and 4 ) is shown to give a stronger signal. Alu, B1 and B2 RT-PCRs were performed with Alu, B1 and B2 consensus primers, respectively (Supplementary Table 1 ).

expression of B1 or B2 transcripts in ES cells (Fig. 5b). Similarly, Alu expression was not increased when HeLa cells were treated with 5-azacytidine (Fig. 5c). In both cases, efficacy of the treatment was confirmed by induction of Apo-E mRNA in the same samples. It was also demonstrated by the release of MBD2 and MeCP2 from B1, B2 and Alu SINEs (Fig. 4a,b). The fact that pol III occupancy did not increase is consistent with the absence of increased expression. Indeed, small but consistent decreases were seen in pol III binding and transcript expression; the reason for this has not been pursued.

PCR with reverse transcription (RT-PCR) does not distinguish between transcripts synthesized by pol III from a SINE's own promoter and pol II-derived RNAs that contain SINE sequences due to the fortuitous presence of these transposable elements in protein-coding genes. We were concerned that a change in pol III-mediated transcription of SINEs might be masked in this assay by constant expression of SINE-containing pol II transcripts. However, the use of a pol III-specific inhibitor established that this is not the case and that the contribution of pol III to the total levels of SINE RNA is detectable using our RT-PCR assay (Supplementary Fig. 9). Nevertheless, primer extension was used to focus on transcripts that initiate specifically at the pol III transcription start site and distinguish these from read-through transcripts initiated upstream ${ }^{6}$. Transcripts detected by this approach are Alu-specific and resistant to doses of $\alpha$-amanitin that inhibit pol II transcription (Supplementary Figs 10,11). Primer extension assays showed that 5-azacytidine treatment makes no significant difference $(P=0.54, t$-test) to the expression of Alu RNA that initiates at the pol III start site, although significantly $(P=0.000065, t$-test $)$ enhancing Apo-E expression (Fig. 5d; Supplementary Fig. 12). We conclude that DNA methylation does not suppress the occupancy or activity of pol III at SINEs, despite attracting MBPs to these loci.

SUV39H1 suppresses pol III occupancy and expression of SINEs. Multiple Alu sequences were recovered in a low-throughput genomic screen of sites bound by K9-methylated histone H3 (ref. 30). Indeed, 68\% of the clones isolated either contained Alu sequence or were $<200 \mathrm{bp}$ from on $\mathrm{e}^{30}$. This constitutes a strong enrichment, since Alu provides $\sim 11 \%$ of genomic DNA. Consistent with this, the Alu loci we tested show clear trimethylation of histone $\mathrm{H} 3$ lysine 9 (H3K9me3; Fig. 6a; Supplementary Fig. 13). In contrast, H3K9me3 is close to background at active $7 S L$ genes.

One of the enzymes that methylates H3K9 is SUV39H1 (ref. 31). That this histone methyltransferase might mediate SINE repression was suggested by the fact that a dominant-negative Suv39h mutant stimulates the expression of B1 and B2 RNA ${ }^{29}$. We detected SUV39H1 at several Alu loci (Alus c10, c19 and c22), but an example was also found (Alu(c6)) where H3K9me3 does not correlate with detection of SUV39H1 (Fig. 6a; Supplementary Fig. 13). SUV39H1 was also detected at the p21 promoter, one of its documented targets ${ }^{32}$, but was close to background at 7SL genes. The combined presence of methylated H3K9 and SUV39H1 has been shown to recruit HP1 (ref. 33). Accordingly, HP1 was detected at the p21 promoter and Alu loci with SUV39H1 and $\mathrm{H} 3 \mathrm{~K} 9 \mathrm{me} 3$, but not at 7SL or Alu(c6) where SUV39H1 is absent. When cells were treated with chaetocin, a selective inhibitor of the SUV39 family ${ }^{34}$, H3K9me3 levels decreased specifically at the Alu sites where SUV39H1 was detected (Fig. 6b; Supplementary Fig. 14). We conclude that SUV39H1 trimethylates $\mathrm{H} 3 \mathrm{~K} 9$ at some Alu loci, but is not unique in this regard. SUV39H1 is one of several H3K9 methyltransferases 35,36 .

At tRNA genes, $\mathrm{H} 3 \mathrm{~K} 9 \mathrm{me} 3$ shows a strong inverse correlation with pol III occupancy ${ }^{37,38}$. Consistent with this, pol III binding to Alu DNA is increased by chaetocin, but only at the loci where H3K9me3 decreases (Fig. 6b; Supplementary Fig. 14). The action of chaetocin is specific, as it increases pol III binding significantly without affecting the binding of TFIIIC or total histone H3. Furthermore, these effects were only observed at loci where SUV39H1 was detected. Elevated Alu expression was observed in chaetocin-treated cells (Fig. 6c) and primer extension demonstrated increased use of the Alu pol III initiation sites (Fig. 6d). The fact that Alu RNA increases when 7SL RNA does not provides evidence that detection of Alu expression is not masked in our assays by the highly abundant 7SL transcript.

The data suggest that $\mathrm{H} 3 \mathrm{~K} 9 \mathrm{me} 3$ is inhibitory to pol III recruitment and therefore contributes to suppression of SINE transcription. To test whether this mark on SINEs depends on methylation of their DNA, we examined its presence in 


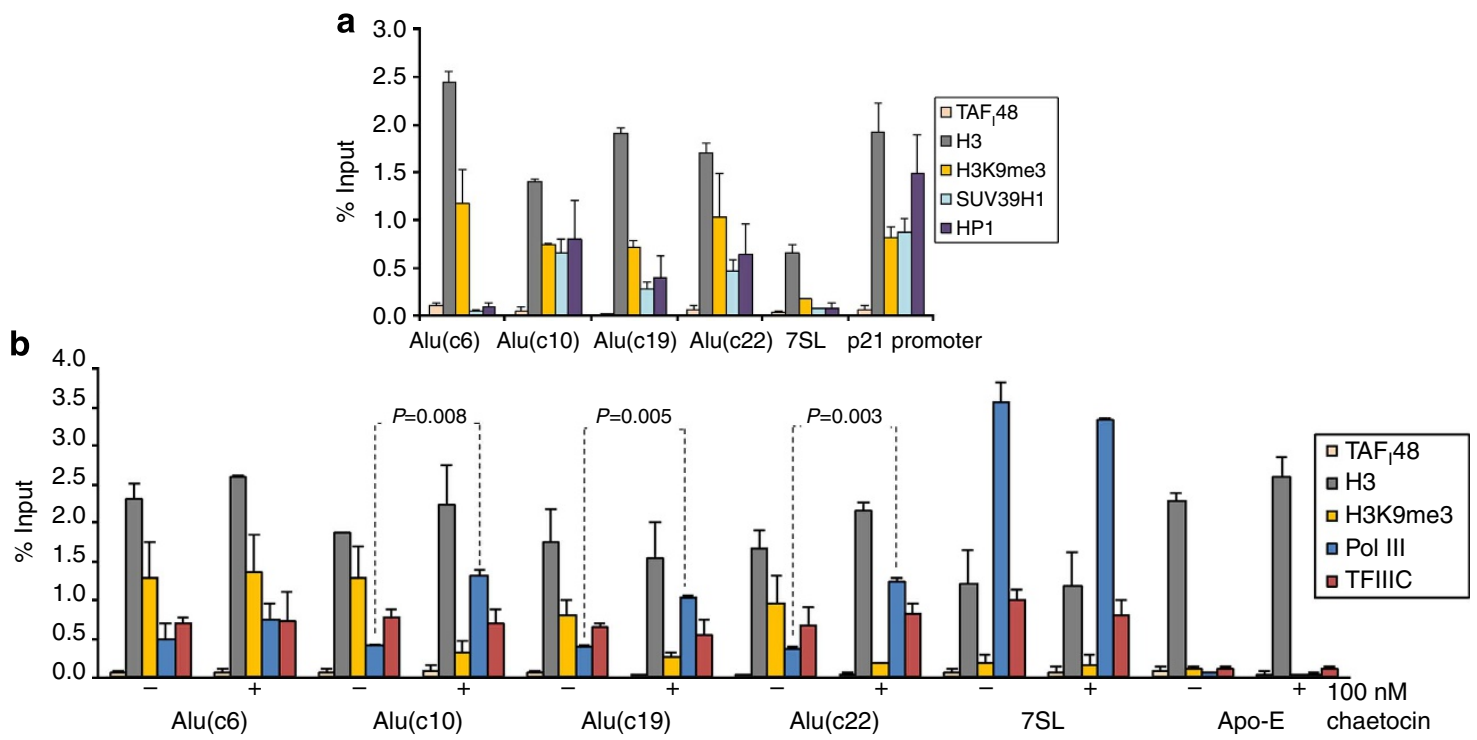

C

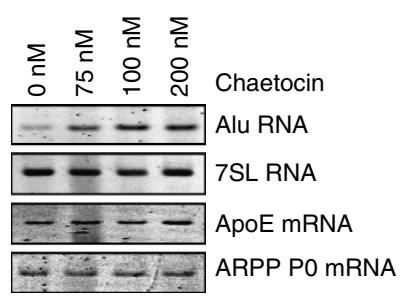

d $075100200 \quad 075100200$ Chaetocin (nM)

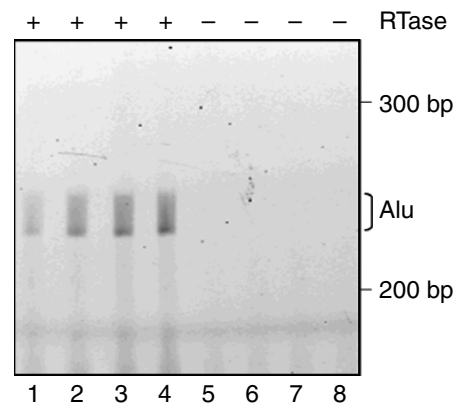

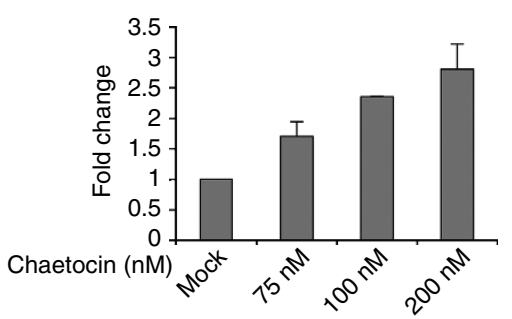

Figure 6 | SUV39H1 inhibits pol III loading and expression of some SINEs. (a) Mean \pm s.e.m. of the percentage input bound in two independent ChIP-quantitative PCR (qPCR) assays with HeLa cells, showing occupancy of H3, TAF,48, H3K9me3, SUV39H1 and HP1 at Alu loci, 7SL and p21 (positive control) genes. (b) Mean \pm s.e.m. of the percentage input bound in two independent ChIP-qPCR assays with HeLa cells treated for $24 \mathrm{~h}$ with vehicle ( - ) or $100 \mathrm{nM}$ chaetocin (+), showing occupancy of H3, TAF 48 , H3K9me3, pol III and TFIIIC at Alu loci, 7SL and Apo-E genes. (c) Semiquantitative RT-PCR analysis of expression levels of indicated transcripts in HeLa cells treated for $24 \mathrm{~h}$ with indicated concentrations of chaetocin. (d) Analysis by primer extension of expression levels of Alu transcripts initiated from pol III start site in HeLa cells treated for $24 \mathrm{~h}$ with indicated concentrations of chaetocin. Reverse transcriptase was omitted from reactions in lanes 5-8. Right panel shows mean \pm s.d. of fold change in Alu expression in two independent experiments quantified using ImageJ. All $P$ values are calculated by $t$-test.

fibroblasts with targeted disruption of Dnmt1. Despite the $>95 \%$ decrease in DNA methylation ${ }^{27}, \mathrm{H} 3 \mathrm{~K} 9 \mathrm{me} 3$ was clearly detected at B1 and B2 SINEs in the Dnmt1-null cells (Fig. 7a). Furthermore, 5 -azacytidine treatment did not significantly decrease the levels of H3K9me3 or SUV39H1 on B1, B2 or Alu SINEs in ES or HeLa cells, although MBD2 binding decreased (Fig. 7b; Supplementary Figs 15,16). In contrast to 5-azacytidine, chaetocin treatment stimulated pol III occupancy and the expression of both B1 and B2 in the presence or absence of Dnmt1 (Fig. 7c,d). These data suggest that SUV39H1 inhibits recruitment of pol III and transcription of SINEs independently of DNA methylation.

DNA methylation suppresses translocation between Alu SINEs. Cytosine methylation has been shown to inhibit homologous recombination between satellite DNA and its deficiency can cause human genetic disease ${ }^{39}$. We therefore considered whether SINE methylation might protect the genome by suppressing DNA rearrangements. This issue is important, because Alu elements are so frequent in the human genome (one every $\sim 3 \mathrm{~kb}$ on average), are concentrated in gene-rich regions and are highly homologous to each other. We tested whether demethylation promotes rearrangements of SINEs using an assay that detects translocations between two Alu copies targeted to chromosomes 14 and 17 in mouse ES cells ${ }^{40}$. Although much less common than recombination between proximal Alu pairs, interchromosomal translocations can be life-threatening ${ }^{1}$. For example, the Alu used in this assay comes from intron 1 of the human $M L L$ gene and participates in MLL duplications that are found in patients with acute myeloid leukaemia ${ }^{41}$. ChIP confirmed that this ectopic Alu is selectively bound by pol III and MBPs in mouse ES cells, as seen for multiple Alus in human cells (Fig. 4a; Supplementary Fig. 17). Translocation is initiated using I-SceI endonuclease to target a double-strand break specifically to the Alu loci. It is detected using a split neomycin phosphotransferase gene (neo) with an MLL Alu positioned downstream of a neo splice donor site on chromosome 17 and another upstream of a neo splice acceptor site on chromosome 14; reciprocal translocations between the two sites generate functional neo ${ }^{+}$genes that can be detected by screening for neomycin-resistance (Fig. 8a). The molecular basis of this resistance was confirmed by detection of translocation-specific PCR products (Supplementary Fig. 18).

Treatment of these cells with 5-azacytidine releases MeCP2 and MBD2 from the MLL-derived Alu elements (Fig. 4a). It also 
a

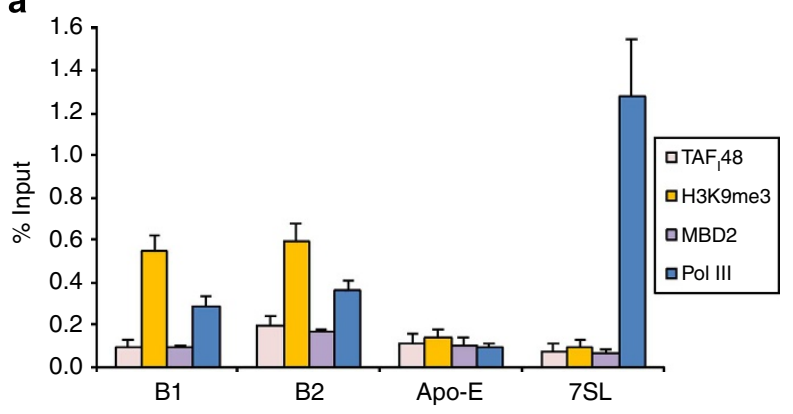

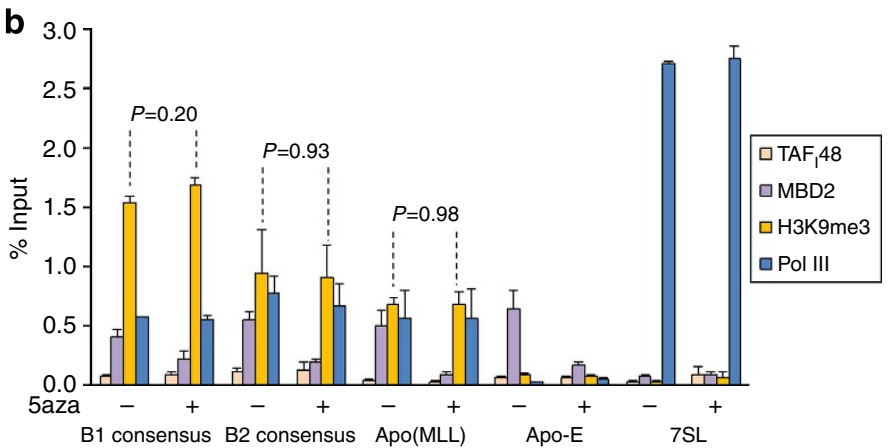
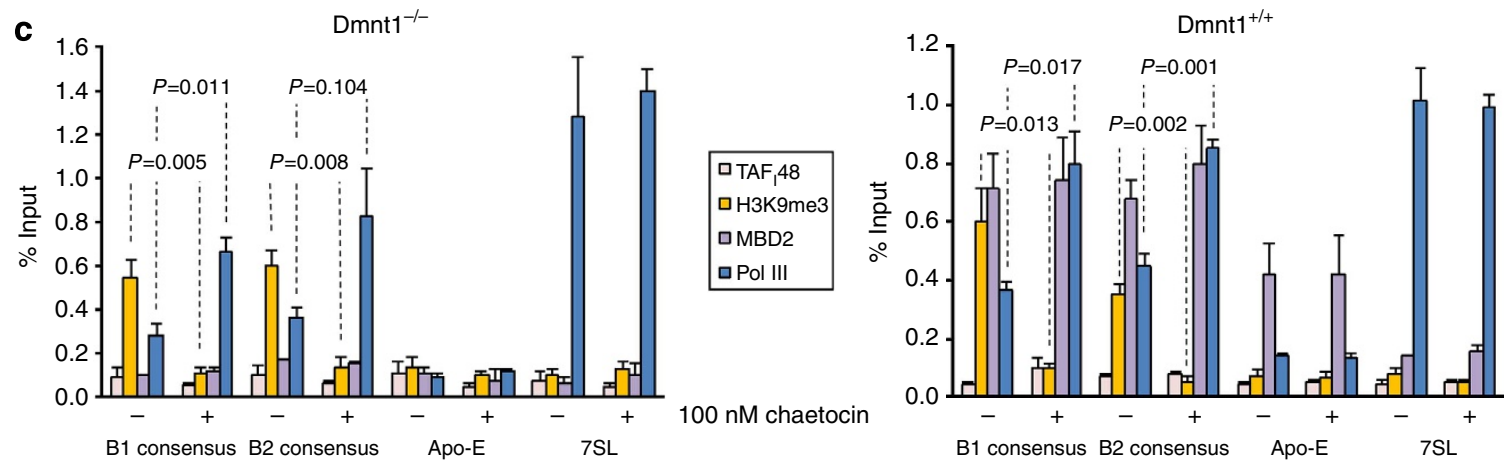

d

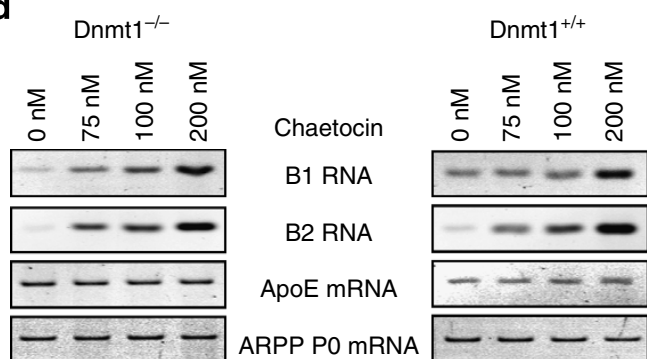

Figure 7 | SINE repression by H3K9Me3 does not require DNA methylation. (a) Percentage input bound in three independent ChIP-quantitative PCR (qPCR) assays with Dnmt1-/- fibroblasts assessing binding of $\mathrm{TAF}_{1} 48, \mathrm{H} 3 \mathrm{~K} 9 \mathrm{me} 3, \mathrm{MBD} 2$ and pol III at 7SL, B1, B2 and Apo-E loci. H3K9me3 data were normalized against total H3. For clarity, Fig. 7a displays a subset of the data shown in Fig. 7c. (b) Percentage input bound in two independent ChIP-qPCR assays of ES cells treated for $16 \mathrm{~h}$ with (+) or without ( - ) 5-azacytidine, assessing binding of TAF,48, MBD2, H3K9me3 and pol III at 7SL, B1 and B2 loci, as well as an Alu inserted onto chromosomes 14 and 17. H3K9me3 data were normalized against total H3. (c) Percentage input bound in three independent ChIP-qPCR assays of matched Dnmt $1^{-/-}$and Dnmt $1^{+/+}$fibroblasts treated for $24 \mathrm{~h}$ with $(+)$ or without $(-) 100 \mathrm{nM}$ chaetocin, assessing binding of $\mathrm{TAF}_{1} 48, \mathrm{H} 3 \mathrm{~K} 9 \mathrm{me} 3, \mathrm{MBD} 2$ and pol III at 7SL, B1 and B2 and Apo-E loci. H3K9me3 data were normalized against total H3. (d) Semiquantitative RT-PCR analysis of expression levels of indicated transcripts in matched Dnmt $1^{+/+}$and Dnmt1 ${ }^{-/}$- fibroblasts treated for $24 \mathrm{~h}$ with indicated concentrations of chaetocin. Error bars indicate s.e.m. and all $P$ values are calculated by $t$-test.

causes a significant increase in translocations (Fig. 8b; Supplementary Data 2). Genomic translocations result from chromosomal breakage and misrepair, especially of double-strand breaks. We therefore tested whether the Alu-mediated translocations are also induced by bleomycin, an agent that induces such breaks efficiently but does not cause demethylation. In contrast to 5azacytidine, bleomycin failed to stimulate Alu translocation in this system. This suggests that the increased rearrangements induced by 5 -azacytidine reflect its demethylating activity.

\section{Discussion}

Retrotransposons provide hotspots for genomic recombination. They contain most of the methylated cytosines in human DNA and this methylation is thought to suppress transposition to promote genomic integrity ${ }^{42}$. Demethylation of satellite DNA has been linked to chromosomal rearrangements and human genetic disease ${ }^{39}$. Because SINEs are concentrated in and around the protein-coding genes, their translocation can be especially disruptive, as illustrated by many oncogenic rearrangements ${ }^{2,43,44}$. For example, acute myeloid leukaemias have arisen through recombination between intronic Alus, leading to partial duplication of the $M L L$ gene ${ }^{45-47}$. Using a model of such lesions, we found that interchromosomal translocation between a pair of Alu SINEs is suppressed by DNA methylation to a significant degree $(P=0.0008, t$-test). SINE-mediated rearrangements may, therefore, contribute to the elevated mutation rates found in most cancer cells where genomic hypomethylation is widespread ${ }^{48-50}$. Indeed, hypomethylation of Alu DNA was found to correlate $(P=0.008)$ with genomic instability in human lung carcinomas ${ }^{51}$.

Translocations between Alus arise when misrepair of a doublestrand break occurs through either of two pathways-nonhomologous end-joining or single-strand annealing ${ }^{40}$. The latter predominates in the assay we have used. This remains the case after treatment with 5-azacytidine (Supplementary Fig. 18), suggesting that demethylation affects the frequency of these 
a
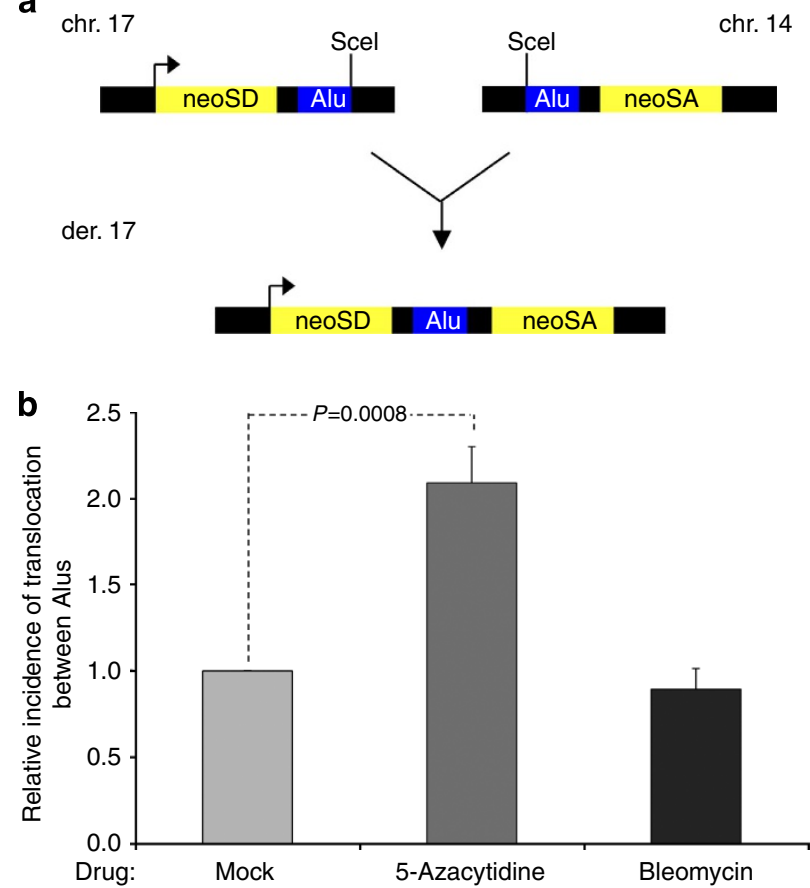

Figure 8 | 5-azacytidine stimulates translocation between Alus.

(a) Schematic illustration of system used to induce and detect chromosomal translocations at Alu elements. (b) Mean \pm s.d. from three independent experiments measuring the relative incidence of translocation between Alu elements located on chromosomes 14 and 17 in ES cells treated with 5 -azacytidine or bleomycin, relative to cells without drug. $P$ values are calculated by $t$-test.

translocations, but not the repair pathway employed. However, demethylation may also influence this assay indirectly by affecting expression of the DNA repair machinery or the rearranged reporter gene. We therefore regard this as an interesting but preliminary observation. Further mechanistic analysis will be required to establish whether methylation of Alu DNA affects rearrangement frequency directly.

Although it may influence translocation, directly or indirectly, SINE DNA methylation does not prevent transcription factor occupancy or function under the conditions we have studied. This was unexpected and contrasts with conclusions drawn from several previous studies. DNA methylation was reported to inhibit TFIIIC binding in vitro, but the DNA fragment tested lacked clear promoter elements ${ }^{52}$. Increased Alu content was found in RNA from lung cancer cell lines after treatment with 5-aza-2'-deoxycytidine, but that study did not distinguish between pol III products and Alu sequences embedded in pol II transcripts ${ }^{51}$. However, the expression of pol III-initiated Alu RNA was shown to be substantially elevated after 8 days treatment of HeLa cells with 5-azacytidine ${ }^{6}$. Indirect effects might have caused this response, given the long duration of drug treatment. The authors confirmed that Alu loci were demethylated $^{6}$, but this does not prove that the Alu demethylation was responsible directly for the observed expression changes. Indeed, a study with $\mathrm{U} 2 \mathrm{OS}$ cells found that pol III transcripts are induced after 5 days treatment with 5 -aza- $2^{\prime}$-deoxycytidine, but not after 2 days; in contrast, the $p 16$ gene, which served as positive control, was induced $>100$-fold in the 2-day-treated samples ${ }^{53}$. Although Alu transcripts were not examined, each of five other pol III products tested showed induction after 5 days but not after 2 days $^{53}$. These data provide clear evidence for secondary effects on the pol III machinery after long-term treatment with demethylating agents. We used shorter drug treatments $(16-72 \mathrm{~h}$ ), which were sufficient to cause SINE demethylation and release of MBPs, but did not enhance expression. Another study found that methylation of a chimeric 7SL/Alu reporter inhibited its expression in transfected cells; contrary to expectation, cotransfection of a MeCP2 overexpression vector relieved this repression, rather than compounding $\mathrm{it}^{17}$. This surprising result may reflect indirect effects or the fact that transiently transfected reporters do not adopt the chromatin structures of endogenous genes ${ }^{54}$. Clear evidence that methylation of Alu DNA can directly inhibit its transcription by pol III has been obtained using assays in vitro ${ }^{13,15}$. However, the inhibitory effect in vitro was only seen at low template concentrations and was absent when more Alu DNA was used ${ }^{13,15}$. Our data suggest that the immunity to repression seen with higher template concentrations mimics the situation in vivo. Nuclear concentrations are orders of magnitude higher than those employed for in vitro transcription reactions. Perhaps, high local concentrations and the context of supercoiled chromatin allow pol III to cope better with methylated templates in situ than in dilute reactions in vitro. The failure of DNA methylation to silence pol III transcription in cells is established here through both genetic and pharmacological approaches. Furthermore, unlike the previous studies, our expression data are supported strongly by direct assessment of pol III occupancy on SINEs and of the methylation state of pol III-bound SINE DNA. The possibility remains, nevertheless, of a more dominant role for DNA methylation in different cell types or conditions.

Although DNA methylation appears not to play a major role in excluding pol III from its templates under the conditions we have studied, trimethylation of $\mathrm{H} 3 \mathrm{~K} 9$ is clearly involved. This modification was already known to be inversely correlated with pol III occupancy and expression of tRNA genes 37 . We found that inhibition of SUV39H1 selectively reduces H3K9 trimethylation and stimulates pol III loading onto a subset of SINEs, while raising expression of SINE transcripts substantially. An exception is Alu(c6), where SUV39H1 inhibition did not significantly alter H3K9me3 or pol III detection (Fig. 6b); this suggests that some SINEs are targeted by $\mathrm{H} 3 \mathrm{~K} 9$ methyltransferases other than SUV39H1. Local environment might dictate which methyltransferase operates at individual SINEs.

In contrast to pol III, the binding of TFIIIC to SINEs is not enhanced by inhibition of SUV39H1 (Fig. 6b). Indeed, TFIIIC detection at SINEs is comparable to that at 7SL genes, which attract much higher levels of pol III (Figs 2a,b and 6b). These observations are consistent with the reports that TFIIIC can overcome chromatin-mediated repression in vitro ${ }^{55,56}$. TFIIIC is responsible for promoter recognition on most pol III-transcribed genes, including SINEs, and then recruits TFIIIB and pol III by protein-protein interactions ${ }^{26,57}$. We believe it is the pol III recruitment step specifically that, rather than promoter recognition, is inefficient in vivo at Alu, B1 and B2 SINEs. Thus, quantification of multiple ChIP experiments revealed a significantly higher ratio of pol III to TFIIIC on 7SL relative to Alu $(P=0.007, t$-test $)$ in human cells and on 7 SL relative to $\mathrm{B} 1$ $(P=0.002, t$-test $)$ and B2 $(P=0.003, t$-test $)$ in mouse cells. Since pol III itself has minimal DNA sequence specificity, it is likely to be the chromatin landscape of SINEs that discourages its recruitment. Our data suggest that $\mathrm{H} 3 \mathrm{~K} 9 \mathrm{me} 3$ is a feature of SINE chromatin that impedes pol III recruitment and transcription.

Despite the inhibitory effects of chromatin, we were able to detect pol III at $\sim 1,400$ Alu SINEs across the human genome, although with generally weaker binding than observed at active tRNA genes. This contrasts with several previous studies that recorded pol III occupancy of very few Alu SINEs $s^{38,58,59}$. For example, only 13 Alu dimer loci were reported as pol III 
bound in ref. 38. The discrepancy may reflect a number of technical differences, including the choice of statistical threshold. Antibody efficiency may be especially important, as the pol III antibody used in our current study gives unusually high enrichment. Using the same pol III antibody as in our current study, Moqtaderi et al. ${ }^{60}$ estimated that $\sim 1,000$ SINEs were pol III occupied in K562 cells, although the levels of pol III at these loci were again generally much lower than at tRNA genes ${ }^{60}$. There is only $\sim 2 \%$ overlap between the sets of Alu loci occupied by pol III in the two studies, which used different cell types. This suggests that access to SINEs may not only be limited, but also highly variable according to conditions. As well as transcriptional suppression, degradation of SINE RNA provides additional protection against accumulation of their transcripts ${ }^{9}$.

\section{Methods}

Cell culture. HeLa cells and A31 fibroblasts were kindly provided by Peter Rigby. Maria Jasin generously provided Hom Alu mouse ES cells, in which an Alu element from intron 1 of the human $M L L$ gene is carried within the Pim 1 locus of chromosome 17 and the $R b$ locus of chromosome 14 (ref. 40). Cells were cultured in DMEM supplemented with $10 \% \mathrm{FCS}, 2 \mathrm{mM}$ L-glutamine, $100 \mathrm{U} \mathrm{ml}^{-1}$ penicillin and $100 \mathrm{U} \mathrm{ml}^{-1}$ streptomycin. For the Dnmt $1^{+9+}$ and Dnmt $1^{-1-}$ fibroblasts, kindly provided by Adrian Bird, Howard Cedar and Ittai Ben-Porath, medium was supplemented with $2 \mathrm{mM}$ sodium pyruvate, $1 \%$ non-essential amino acids and $0.01 \% \beta$-mercaptoethanol; these cells have a p53-null background ${ }^{27}$. 5-azacytidine (Sigma) was used at $4 \mu \mathrm{M}$ for $16-72 \mathrm{~h}$, as indicated. Bleomycin (Calbiochem) was used at $5 \mu \mathrm{g} \mathrm{ml}^{-1}$ for $16 \mathrm{~h}$. Treatment with chaetocin (Sigma) was for $24 \mathrm{~h}$ and with $\alpha$-amanitin (Sigma) was as indicated.

ChIP assays. ChIP-PCR and sequential ChIP-PCR assays were performed as previously described ${ }^{61}$. Protein-DNA complexes were cross-linked using $1 \%$ formaldehyde for $10 \mathrm{~min}$ at room temperature and the reaction was quenched with $0.125 \mathrm{M}$ glycine. Cells were washed with PBS $0.5 \% \mathrm{NP}-40$ and lysed by incubation in $1 \mathrm{M} \mathrm{NaCl} \mathrm{PBS} / \mathrm{NP}-40$, followed by $0.1 \mathrm{M} \mathrm{NaCl}$ and $10 \mathrm{mM}$ TE/NP-40. The nuclei were then cleaned by centrifugation through a $100 \mathrm{mM}$ sucrose cushion. The chromatin was sheared by water bath sonication using a Bioruptor (Diagenode) to obtain a median fragment size of $500 \mathrm{bp}$. Input samples were removed and immunoprecipitation was performed overnight in $150 \mathrm{mM} \mathrm{NaCl} \mathrm{TE} / \mathrm{NP}-40$ using $5 \mu \mathrm{g}$ antibody bound to protein $\mathrm{A} / \mathrm{G}$ sepharose. Immunoprecipitates were washed twice in RIPA buffer, twice in $250 \mathrm{mM} \mathrm{LiCl} 0.5 \% \mathrm{Na}$-deoxycholate TE/NP-40, twice in TE and elution was performed in TE/1\%SDS. The eluate from the first ChIP was diluted 1:10 before a sequential ChIP. Proteinase K digestion was performed overnight at $42^{\circ} \mathrm{C}$. DNA was purified with QIAquick PCR purification kit (Qiagen), according to the manufacturers' instructions.

Serial dilutions of the input chromatin were used to confirm that PCRs were within a linear range. $P$ values were obtained using a two-tailed unpaired $t$-test. PCR primers and amplification conditions are described in Supplementary Table 1. Individual loci were specified using primers for unique sequence flanking a particular SINE. The B1(c9) and B2(c9) primers detect unique sequences on $\mathrm{c} 9$ within a cluster that has no recognized pol II transcription unit within $5 \mathrm{~kb}$. B1 and B2 primers detect subgroups of $\sim 10^{2}$ family members each. The Alu consensus primers match the PV subfamily that has $\sim 10^{3}$ members.

For ChIP-chop assay ${ }^{24}$, ChIP DNA was spiked with $100 \mathrm{ng}$ of unmethylated PCR product containing a HpaII/MspI site (to normalize for digestion efficiency) and then digested for $1 \mathrm{~h}$ with HpaII or MspI.

Antibodies used were M9317 against MeCP2 and M7318 against MBD2 (Sigma), IMG-306A against MBD1 (Imgenex), ab1791 against histone H3 (Abcam), 07-108 against histone H4 and 05-615 against SUV39H1 (Upstate), 9754S against H3K9me3 (Cell Signaling), sc-25366 against SUV39H1, sc-28735 against HP1, sc-25365 against TFIIA and sc-6571 against TAF $_{1} 48$ (Santa Cruz Biotechnology), 1900 against the RPC155 subunit of Pol III' ${ }^{62}, 128$ against the Brf1 subunit of TFIIIB, 4286 against the $110 \mathrm{kD}$ subunit of TFIIIC ${ }^{63}$ and Ab7 against the $220 \mathrm{kD}$ subunit of TFIIIC ${ }^{37}$. Uncropped scans are shown in Supplementary Fig. 19.

ChIP-BS-Seq library preparation and sequencing. For HeLa input DNA, two libraries were prepared from the same input DNA: one with starting quantity of $10 \mathrm{ng}$, following the Illumina ChIP-Seq library protocol, with the addition of bisulfite conversion after addition of adaptors but before amplification; and one following the Illumina Bisulfite Sequencing protocol, including the recommended starting amount. All libraries included addition of $2 \%$ sheared lambda DNA to control for bisulfite conversion. The Qiagen EpiTect kit was used for bisulfite conversion, with four periods of incubation at $60^{\circ} \mathrm{C}$ (for $25,85,175$ and $120 \mathrm{~min}$ ), each preceded by denaturation for $10 \mathrm{~min}$ at $98^{\circ} \mathrm{C}$. Size selection was not performed for the amplified libraries (except the regular input library) due to the small insert size and the small total amount of total library after amplification. The first library was subjected to 101-cycle paired-end sequencing (one lane) and the second library was subjected to 101-cycle single-end sequencing (one lane) on the Illumina HiSeq. For pol III ChIP-BS-Seq, one library was prepared with eluate from 10 technical replicate RPC155 ChIP assays carried out as previously ${ }^{38}(\sim 10 \mathrm{ng}$; all replicates were from the same batch of cross-linked HeLa cells, grown to $75 \%$ confluence), following the Illumina ChIP-Seq library protocol with bisulfite conversion as for the first input library. The pol III library was subjected to 101-cycle single-end sequencing (three lanes) on the Illumina HiSeq.

ChIP-BS-Seq analysis. A novoalign index was created for the hg18 genome (UCSC), plus the Lambda genome (Genbank accession number J02459.1) and adapter sequences (Illumina PE PCR Primer 2.0) with these options: novoindex $-k$ 13 -s 3 -b. Reads were aligned with novoalign using paired-end (-FILMFQ -t120 -h120 -b2 -a -i PE 20-600) or single-end settings (-FILMFQ -t120 -h120 -b2 -a). Repeat alignments intersecting at least one uniquely aligned sequence realigned using single-end settings, allowing up to seven alignments (-r A 7), which includes $85 \%$ of the repeat reads. For input, we obtained 94122299 genomic alignments (86740371 uniquely aligned reads and 2491119 reads with 2-7 alignments); for RPC155, we obtained 43883712 genomic alignments (37007535 uniquely aligned reads and 2694788 reads with 2-7 alignments). In addition, 1344324 reads of the input library and 1187215 reads of the pol III library aligned to the Lambda genome. The rates of conversion of methylated cytosines in the reads aligning to Lambda are $99.87 \%$ and $99.64 \%$ for input and pol III libraries, respectively.

Analysis of aligned data was performed with the USeq package of programs (useq.sourceforce.net). For ChIP-Seq analysis, alignments were converted to singleposition point data with NovoalignParser (with no quality filter, to include repeat alignments) and peaks were called with ScanSeqs (using peakshift of $116 \mathrm{bp}$, as determined by PeakShiftFinder, and $300 \mathrm{bp}$ window) and EnrichedRegionMaker with thresholds of 20 or 70,13 and 1 for $Q$-value false discovery rate (FDR), empirical FDR and $\log 2$ ratio, respectively. To obtain a list of enriched pol IIItranscribed genes (including SINEs), pol III-enriched regions ( $Q$-value 20 threshold) were intersected with an annotation file containing all SINEs, tRNA genes and fragments, and other pol III-transcribed genes and elements from the RepeatMasker track of UCSC. The annotated genes/repeat elements were scored by DefinedRegionScanSeqs using the PointData and $Q$-value 70 threshold was used. The number of enriched genes by class is 1,472 Alu, 242 tRNA, 83 MIR, seven U6, four hY, three 7SL/SRP, three HVG, two 5S rRNA, two miRNA, one RNaseP, one MRP, one $\mathrm{BC} 200$ and one $7 \mathrm{SK}$ to a total of 1,822 genes.

For methylation analysis, alignments were converted to per-base methylated and unmethylated cytosines with NovoalignBisulfitParser (with no quality filter to include repeat alignments). Regions statistically enriched or reduced for methylation were determined with BisSeq (using settings -w 10 -m 10 -f 13 -1 0). Percent methylation scores for enriched pol III target genes (including SINEs) were obtained using ScoreMethylatedRegions.

To compare pol III enrichment scores and methylation fractions between Alu families (for example, AluJ, AluS and AluY), a non-parametric Kruskal-Wallis test was performed, followed by Dunn's multiple comparison test.

For alignment of reads to the Alu consensus sequences, Alu sequences were obtained from Repbase and concatenated with 100xN separating the sequences. This fasta file was indexed with novoindex. Genomic reads were filtered for those intersecting with SINEs, converted to fastq format and realigned to the Alu consensus index with novoalign (-r All 14 -t240 -h120 -b2 -a -s). Per-base cytosine methylation graphs were obtained with BisStat and visualized with IGB (www.bioviz.org).

Affinity chromatography. Separation of genomic DNA according to CpG methylation status was achieved by affinity chromatography with immobilized recombinant MBD2b and MBD3L1 using a MethylCollector Ultra (Active Motif), according to the manufacturer's specifications.

Gene expression analysis. To synthesize complementary DNA for RT-PCR, SuperScript III reverse transcriptase (Invitrogen) was used as previously ${ }^{61}$, with $200 \mathrm{ng}$ of RNA and Hexanucleotide Mix (Roche). Primers and amplification conditions are described in Supplementary Table 1. Primer extension was performed using 5-carboxyfluorescein end-labelled (Invitrogen) Alu 21mer primer. RNA $(5-10 \mu \mathrm{g})$ was denatured with $100 \mathrm{ng}$ of labelled probe in $20 \mu \mathrm{l}$ of $1 \times$ First Strand Buffer (Invitrogen) at $80^{\circ} \mathrm{C}$ for $10 \mathrm{~min}$. Primer annealing was performed at $56^{\circ} \mathrm{C}$ for $2 \mathrm{~h}$. Thirty microlitre of elongation mix containing $100 \mathrm{U}$ of SuperScript III (Invitrogen), 1:50 RNAsin (Promega), $2 \mathrm{mM}$ dithiothreitol, $1 \mathrm{mM}$ dNTP and $10 \mathrm{ng}^{-1}$ actinomycin D (Sigma) were added and samples were incubated at $42{ }^{\circ} \mathrm{C}$ for $1 \mathrm{~h}$. Nucleic acids were precipitated in $0.1 \mathrm{M} \mathrm{NaOAc}$ and ethanol overnight with $1 \mu \mathrm{l}$ of $1 \mathrm{M}$ purified yeast tRNA as carrier. Samples were resolved on $7 \%$ sequencing gels and visualized using Typhoon 9400 (GE Healthcare). All $P$ values were obtained using a two-tailed unpaired $t$-test.

Translocation assay. Culture of Hom Alu mouse ES cells and translocation assays were conducted as previously ${ }^{40}$. Cells $\left(10^{7}\right)$ were electroporated with $25 \mu \mathrm{g}$ of pTK-hyg or pCBAS and were allowed to recover from electroporation for $10 \mathrm{~h}$ before treatment for $16 \mathrm{~h}$ with 5 -azacytidine or bleomycin. Cells were then allowed to recover in growth medium for $24 \mathrm{~h}$ and then subjected to selection with 
$200 \mu \mathrm{g} \mathrm{ml}^{-1}$ neomycin for 8-10 days. A plate without selection was used to calculate the loss of cell viability due to drug treatment. Colonies obtained after selection were GIEMSA stained for counting or picked for PCR analysis.

\section{References}

1. Batzer, M. A. \& Deininger, P. L. Alu repeats and human genomic diversity. Nat. Rev. Genet. 3, 370-379 (2002).

2. Burns, K. H. \& Boeke, J. D. Human transposon tectonics. Cell 149, 740-752 (2012).

3. Nicholls, R. D., Fischel-Ghodsian, N. \& Higgs, D. R. Recombination at the human alpha-globin gene cluster: sequence features and topological constraints. Cell 49, 369-378 (1987).

4. Lehrman, M. A., Goldstein, J. L., Russell, D. W. \& Brown, M. S. Duplication of seven exons in LDL receptor gene caused by Alu-Alu recombination in a subject with familial hypercholesterolemia. Cell 48, 827-835 (1987).

5. Wallace, M. R. et al. A de novo Alu insertion results in neurofibromatosis type 1. Nature 353, 864-866 (1991).

6. Liu, W. M., Maraia, R. J., Rubin, C. M. \& Schmid, C. W. Alu transcripts: cytoplasmic localisation and regulation by DNA methylation. Nucleic Acids Res. 22, 1087-1095 (1994).

7. Russanova, V. R., Driscoll, C. T. \& Howard, B. H. Adenovirus type 2 preferentially stimulates polymerase III transcription of Alu elements by relieving repression: a potential role for chromatin. Mol. Cell Biol. 15, 4282-4290 (1995).

8. Ponicsan, S. L., Kugel, J. F. \& Goodrich, J. A. Genomic gems: SINE RNAs regulate mRNA production. Curr. Opin. Genet. Dev. 20, 149-155 (2010).

9. Kaneko, H. et al. DICER1 deficit induces Alu RNA toxicity in age-related macular degeneration. Nature 471, 325-330 (2011).

10. Miranda, T. B. \& Jones, P. A. DNA methylation: the nuts and bolts of repression. J. Cell Physiol. 213, 384-390 (2007).

11. Suzuki, M. M. \& Bird, A. DNA methylation landscapes: provocative insights from epigenomics. Nat. Rev. Genet. 9, 465-476 (2008).

12. Xie, H. et al. Epigenomic analysis of Alu repeats in human ependymomas. Proc. Natl Acad. Sci. USA 107, 6952-6957 (2010).

13. Liu, W. M. \& Schmid, C. W. Proposed roles for DNA methylation in Alu transcriptional repression and mutational inactivation. Nucleic Acids Res. 21, 1351-1359 (1993).

14. Schmid, C. W. Human Alu subfamilies and their methylation revealed by blot hybridization. Nucleic Acids Res. 19, 5613-5617 (1991).

15. Kochanek, S., Renz, D. \& Doerfler, W. DNA methylation in the Alu sequences of diploid and haploid primary human cells. EMBO J. 12, 1141-1151 (1993).

16. Meissner, A. et al. Genome-scale DNA methylation maps of pluripotent and differentiated cells. Nature 454, 766-770 (2008).

17. Yu, F., Zingler, N., Schumann, G. \& Stratling, W. H. Methyl-CpG-binding protein 2 represses LINE-1 expression and retrotransposition but not Alu transcription. Nucleic Acids Res. 29, 4493-4501 (2001).

18. Bird, A. P. \& Wolffe, A. P. Methylation-induced repression--belts, braces, and chromatin. Cell 99, 451-454 (1999).

19. Koch, C. \& Stratling, W. H. DNA binding of methyl-CpG-binding protein MeCP2 in human MCF7 cells. Biochemistry 43, 5011-5021 (2004).

20. Ballestar, E. et al. Methyl-CpG binding proteins identify novel sites of epigenetic inactivation in human cancer. EMBO J. 22, 6335-6345 (2003).

21. Ullu, E. \& Tschudi, C. Alu sequences are processed 7SL RNA genes. Nature 312, 171-172 (1984).

22. Waterston, R. H. et al. Initial sequencing and comparative analysis of the mouse genome. Nature 420, 520-562 (2002).

23. Daniels, G. R. \& Deininger, P. L. Repeat sequence families derived from mammalian tRNA genes. Nature 317, 819-822 (1985).

24. Lawrence, R. J. et al. A concerted DNA methylation/histone methylation switch regulates rRNA gene dosage control and nucleolar dominance. Mol. Cell 13, 599-609 (2004).

25. Brinkman, A. B. et al. Sequential ChIP-bisulfite sequencing enables direct genome-scale investigation of chromatin and DNA methylation cross-talk. Genome Res. 22, 1020-1027 (2012).

26. Schramm, L. \& Hernandez, N. Recruitment of RNA polymerase III to its target promoters. Genes Dev. 16, 2593-2620 (2002).

27. Jorgensen, H. F., Ben-Porath, I. \& Bird, A. P. Mbd1 is recruited to both methylated and nonmethylated CpGs via distinct DNA binding domains. Mol. Cell Biol. 24, 3387-3395 (2004).

28. Sarraf, S. A. \& Stancheva, I. Methyl-CpG binding protein MBD1 couples histone $\mathrm{H} 3$ methylation at lysine 9 by SETDB1 to DNA replication and chromatin assembly. Mol. Cell 15, 595-605 (2004).

29. Martens, J. H. et al. The profile of repeat-associated histone lysine methylation states in the mouse epigenome. EMBO J. 24, 800-812 (2005).

30. Kondo, Y. \& Issa, J. P. Enrichment for histone H3 lysine 9 methylation at Alu repeats in human cells. J. Biol. Chem. 278, 27658-27662 (2003).

31. Rea, S. et al. Regulation of chromatin structure by site-specific histone $\mathrm{H} 3$ methyltransferases. Nature 406, 593-599 (2000).
32. Cherrier, T. et al. p21(WAF1) gene promoter is epigenetically silenced by CTIP2 and SUV39H1. Oncogene 28, 3380-3389 (2009).

33. Stewart, M. D., Li, J. \& Wong, J. Relationship between histone H3 lysine 9 methylation, transcription repression, and heterochromatin protein 1 recruitment. Mol. Cell Biol. 25, 2525-2538 (2005).

34. Greiner, D., Bonaldi, T., Eskeland, R., Roemer, E. \& Imhof, A. Identification of a specific inhibitor of the histone methyltransferase SU(VAR)3-9. Nat. Chem. Biol. 1, 143-145 (2005).

35. Tachibana, M. et al. G9a histone methyltransferase plays a dominant role in euchromatic histone H3 lysine 9 methylation and is essential for early embryogenesis. Genes Dev. 16, 1779-1791 (2002).

36. Lakshmikuttyamma, A., Scott, S. A., DeCoteau, J. F. \& Geyer, C. R. Reexpression of epigenetically silenced AML tumor suppressor genes by SUV39H1 inhibition. Oncogene 29, 576-588 (2010).

37. Barski, A. et al. Pol II and its associated epigenetic marks are present at Pol IIItranscribed noncoding RNA genes. Nat. Struct. Mol. Biol. 17, 629-634 (2010).

38. Oler, A. J. et al. Human RNA polymerase III transcriptomes and relationships to Pol II promoter chromatin and enhancer-binding factors. Nat. Struct. Mol. Biol. 17, 620-628 (2010).

39. Xu, G. L. et al. Chromosome instability and immunodeficiency syndrome caused by mutations in a DNA methyltransferase gene. Nature 402, 187-191 (1999).

40. Elliott, B., Richardson, C. \& Jasin, M. Chromosomal translocation mechanisms at intronic alu elements in mammalian cells. Mol. Cell 17, 885-894 (2005).

41. Hess, J. L. MLL: a histone methyltransferase disrupted in leukemia. Trends Mol. Med. 10, 500-507 (2004).

42. Yoder, J. A., Walsh, C. P. \& Bestor, T. H. Cytosine methylation and the ecology of intragenomic parasites. Trends Genet. 13, 335-340 (1997).

43. Nystrom-Lahti, M. et al. Founding mutations and Alu-mediated recombination in hereditary colon cancer. Nat. Med. 1, 1203-1206 (1995).

44. Kolomietz, E., Meyn, M. S., Pandita, A. \& Squire, J. A. The role of Alu repeat clusters as mediators of recurrent chromosomal aberrations in tumors. Genes Chromosomes Cancer 35, 97-112 (2002).

45. Schichman, S. A. et al. ALL-1 tandem duplication in acute myeloid leukemia with a normal karyotype involves homologous recombination between Alu elements. Cancer Res. 54, 4277-4280 (1994).

46. So, C. W. et al. MLL self fusion mediated by Alu repeat homologous recombination and prognosis of AML-M4/M5 subtypes. Cancer Res. 57, 117-122 (1997).

47. Strout, M. P., Marcucci, G., Bloomfield, C. D. \& Caligiuri, M. A. The partial tandem duplication of ALL1 (MLL) is consistently generated by Alu-mediated homologous recombination in acute myeloid leukemia. Proc. Natl Acad. Sci. USA 95, 2390-2395 (1998).

48. Feinberg, A. P. \& Vogelstein, B. Hypomethylation distinguishes genes of some human cancers from their normal counterparts. Nature 301, 89-92 (1983).

49. Baylin, S. \& Bestor, T. H. Altered methylation patterns in cancer cell genomes: cause or consequence? Cancer Cell 1, 299-305 (2002).

50. Jones, P. A. \& Baylin, S. B. The fundamental role of epigenetic events in cancer. Nat. Rev. Genet. 3, 415-428 (2002).

51. Daskalos, A. et al. Hypomethylation of retrotransposable elements correlates with genomic instability in non-small cell lung cancer. Int. J. Cancer 124, 81-87 (2009).

52. Bartke, T. et al. Nucleosome-interacting proteins regulated by DNA and histone methylation. Cell 143, 470-484 (2010).

53. Selvakumar, T., Gjidoda, A., Hovde, S. L. \& Henry, R. W. Regulation of human RNA polymerase III transcription by DNMT1 and DNMT3a DNA methyltransferases. J. Biol. Chem. 287, 7039-7050 (2012).

54. Jeong, S. \& Stein, A. Micrococcal nuclease digestion of nuclei reveals extended nucleosome ladders having anomalous DNA lengths for chromatin assembled on non-replicating plasmids in transfected cells. Nucleic Acids Res. 22, 370-375 (1994).

55. Burnol, A. F. et al. TFIIIC relieves repression of U6 snRNA transcription by chromatin. Nature 362, 475-477 (1993).

56. Kundu, T. K., Wang, Z. \& Roeder, R. G. Human TFIIIC relieves chromatinmediated repression of RNA polymerase III transcription and contains an intrinsic histone acetyltransferase activity. Mol. Cell Biol. 19, 1605-1615 (1999).

57. Geiduschek, E. P. \& Kassavetis, G. A. The RNA polymerase III transcription apparatus. J. Mol. Biol. 310, 1-26 (2001).

58. Canella, D., Praz, V., Reina, J. H., Cousin, P. \& Hernandez, N. Defining the RNA polymerase III transcriptome: genome-wide localization of the RNA polymerase III transcription machinery in human cells. Genome Res. 20, 710-721 (2010).

59. Raha, D. et al. Close association of RNA polymerase II and many transcription factors with Pol III genes. Proc. Natl Acad. Sci. USA 107, 3639-3644 (2010).

60. Moqtaderi, Z. et al. Genomic binding profiles of functionally distinct RNA polymerase III transcription complexes in human cells. Nat. Struct. Mol. Biol. 17, 635-640 (2010).

61. Kantidakis, T. \& White, R. J. Dr1 (NC2) is present at tRNA genes and represses their transcription in human cells. Nucleic Acids Res. 38, 1228-1239 (2009). 
62. Fairley, J. A., Scott, P. H. \& White, R. J. TFIIIB is phosphorylated, disrupted and selectively released from tRNA promoters during mitosis in vivo. EMBO J. 22, 5841-5850 (2003).

63. Sutcliffe, J. E., Brown, T. R., Allison, S. J., Scott, P. H. \& White, R. J. Retinoblastoma protein disrupts interactions required for RNA polymerase III transcription. Mol. Cell Biol. 20, 9192-9202 (2000).

\section{Acknowledgements}

We thank Adrian Bird, Howard Cedar and Ittai Ben-Porath for matched Dnmt1 ${ }^{+1+}$ and Dnmt1 ${ }^{-1-}$ fibroblasts and Maria Jasin for Hom Alu cells. We are grateful to Patricia Sung for the advice on using the Hom Alu cells. We also thank David Gillespie, Irina Stancheva, Adrian Bird and Peter Jones for valuable advice and discussion. This work was funded by studentships from the Wellcome Trust (073424/Z/03/Z) and Cancer Research UK (for R.J.W. lab) and by HHMI and the Huntsman Cancer Institute (for B.R.C. lab).

\section{Author contributions}

D.V., J.V-A. and A.J.O. carried out the experiments and data analysis. V.H.C. provided the advice, lab space and reagents to allow D.V. to address referee's comments. B.R.C and A.J.O. designed and interpreted the ChIP-BS-Seq analysis. R.J.W. conceived the study, supervised D.V. and J.V-A. and wrote the manuscript.

\section{Additional information}

Accession codes: ChIP-BS-Seq data deposited in the NCBI Gene Expression Omnibus database under GEO accession number GSE38794.

Supplementary Information accompanies this paper at http://www.nature.com/ naturecommunications

Competing financial interests: There are no competing financial interests.

Reprints and permission information is available online at http://npg.nature.com/ reprintsandpermissions/

How to cite this article: Varshney, D. et al. SINE transcription by RNA polymerase III is suppressed by histone methylation but not DNA methylation. Nat. Commun. 6:6569 doi: 10.1038/ncomms7569 (2015)

(c) (i) This work is licensed under a Creative Commons Attribution 4.0 International License. The images or other third party material in this article are included in the article's Creative Commons license, unless indicated otherwise in the credit line; if the material is not included under the Creative Commons license, users will need to obtain permission from the license holder to reproduce the material. To view a copy of this license, visit http://creativecommons.org/licenses/by/4.0/ 